\title{
STUDY OF MATERNAL BMI AT TERM PREGNANCY AND PREGNANCY OUTCOME
}

\section{Dr. B. Goswami}

\section{Dr. S.S.} Choudhury*

\section{Dr. Priyanka. N}

Assistant Professor, Gauhati Medical College and Hospital, Bhangagarh, Guwahati, Assam 781032, India.

Associate Professor, Gauhati Medical College and Hospital, Bhangagarh, Guwahati, Assam 781032, India. *Corresponding Author

Post Graduate Trainee, Gauhati Medical College and Hospital, Bhangagarh, Guwahati, Assam 781032, India.

ABSTRACT Background: The health and quality of life of a pregnant women and her baby is determined by the nutritional status. The risks of extremes of maternal nutritional status and the related pregnancy complications is a growing problem. Maternal surveillance can be done better with the knowledge of maternal BMI and it helps in the prevention of complications. Aim: To determine the association between maternal BMI at term pregnancy and its antenatal maternal complications, labour and neonatal complications and to evaluate the risk of developing adverse maternal and foetal outcomes in women with extremes of BMI. Study design: Prospective observational study. Materials And Methods: 1000 antenatal women with singleton live pregnancies within 37-42 weeks of gestational age attending ANOPD or ELR were included to study the effect of maternal BMI at term pregnancy and pregnancy outcomes. Results: Obese women had the highest incidence and highest risk of developing antenatal complications like GDM (7.6\%) ( $p=0.02)$, PIH $(17.6 \%)(p<0.0001)$, preeclampsia $(12.8 \%)(p<0.01)$ and labour outcomes such as instrumental vaginal deliveries $(9.3 \%)(p<0.05)$, LSCS $(52.3 \%)$ $(p<0.0001)$ and early puerperal outcomes such as PPH $(18.1 \%)(p=0.0002)$ and delayed wound healing $(10.4 \%)(p=0.0006)$. Obese women also had the highest incidence and highest risk of neonatal outcomes such as LGA babies $(12 \%)(p=0.0002)$ and NICU admission (11.9\%) ( $p=0.01$ ). Underweight women had the highest incidence and highest risk of anaemia $(57.3 \%)$ $(p<0.0001)$ and delivering SGA babies $(21 \%)(p<0.0001)$. Conclusion: The present study establishes the adverse maternal and neonatal outcomes associated with the extremes of maternal BMI. This study emphasizes the importance of the nutritional status and health of mothers which helps to reduce the adverse outcomes.

\section{KEYWORDS : maternal BMI, pregnancy, underweight, obesity, adverse foetal and maternal outcomes}

\section{INTRODUCTION:}

The health of women during her pregnancy, childbirth and postnatal period is referred as maternal health. WHO recommends that every woman should be provided with antenatal care which provides health care functions including health promotion, screening, diagnosis and disease prevention. [1]

Undernutrition in pregnancy is associated with anaemia and toxaemias of pregnancy in the mother and adverse neonatal outcomes such as IUGR, increased infant hospitalization rate with higher risk of cognitive and mental impairment and death. [2]

Overweight and obese women are more prone for miscarriage, foetal anomalies, gestational diabetes, gestational hypertension, preeclampsia, venous thromboembolism, hyperlipidaemia, prolonged pregnancies, failed induction, increased frequency of caesarean section, anaesthetic complications like failed regional blocks and difficult intubation, operative vaginal delivery, macrosomia, shoulder dystocia, stillbirth, 3rd and 4th degree perineal lacerations and increased risk of postpartum haemorrhage [3]. Postnatal complications include increased postoperative morbidity rate like urine, uterine and wound infections, reduced breastfeeding success rates, prolonged hospital stay. The adverse neonatal outcomes are LGA babies, lower APGAR score, gross congenital malformations, increased NICU admission rates and IUFD.

Body mass index or Quetelet's index is a statistical index that provides an estimate of body fat in males and females of any age using a person's weight and height. [4] It is calculated using the formula

BMI $=$ Weight $(\mathrm{kgs}) /$ Height $(\mathrm{m} 2)$

BMI is universally accepted and the most commonly used anthropometric analysis due to its reliability and simplicity of the equation. It is used to quantify the tissue mass present in a person and as a categorizing factor of body fatness. Maternal surveillance can be done better with the knowledge of maternal BMI and it helps in the prevention of complications.

\section{MATERIALS AND METHODS:}

This prospective observational study comprising 1000 antenatal women was conducted in the Deptt. of Obstetrics and Gynaecology, Gauhati Medical College, Guwahati, India, from lst June, 2019 to 31st May, 2020 to study the effect of maternal BMI at term pregnancy and pregnancy outcomes. This study was done after taking approval from Institutional Ethical Committee. They were informed about the study and consent was taken. Detailed history was taken regarding name, age, area of residence and socioeconomic status. Obstetric history, past medical and surgical history, family history was noted. General and systemic examination was done.

Patients Were Divided Into 4 Groups Based On Their BMI

- Underweight: $<18.5 \mathrm{~kg} / \mathrm{m} 2$

- Normal weight: $18.5-24.9 \mathrm{~kg} / \mathrm{m} 2$

- Overweight: $25-29.9 \mathrm{~kg} / \mathrm{m} 2$

- Obese: $>30 \mathrm{~kg} / \mathrm{m} 2$

Inclusion Criteria:

- Singleton live pregnancy

- Patients at term gestation (37-42 weeks)

Exclusion Criteria:

1) Patients With Pre-existing Medical Disorders Such As

- Chronic hypertension

- Overt diabetes

- Overt hypothyroidism/hyperthyroidism

- Connective tissue disorders like SLE

- Heart disease 
- Kidney disorders

2) Patients on long term drugs causing weight gain: OCPs, antipsychotics, corticosteroids

\section{3) Multiple Gestation}

Information regarding complications in mother, labour outcomes, birth weight and NICU admission of neonate were collected from case sheets following delivery. Results were analysed to study the association and relative risk between maternal BMI and adverse maternal, labour and neonatal outcomes.

\section{RESULTS:}

The outcomes were statistically analysed and the following results were obtained. The age distribution of the study population was $18-38$ years with a mean age of 27 years. $9 \%$ in $\leq 20$ years, $29.7 \%$ in $21-25$ years, $39.7 \%$ in $26-30$ years, $15.3 \%$ in $31-35$ years, $6.3 \%$ in $>35$ years age group. $556(55.6 \%)$ were primigravida and $444(44.4 \%)$ were multigravida. Distribution of patients based on BMI: underweight - 144(14.4\%), normal weight-532(53.2\%), overweight-238(23.8\%), obese-86(8.6\%).

Table 1: Table Showing The Incidence And Relative Risk Of Antenatal Complications In Various Categories Of BMI:

\begin{tabular}{|l|l|l|l|l|l|l|l|l|}
\hline OUTCOMES & UNDER WEIGHT & RR & NORMAL WEIGHT & OVER WEIGHT & RR & OBESE & RR & p Value \\
\hline GDM & $3(2.1 \%)$ & 0.8 & $13(2.4 \%)$ & $12(5.1 \%)$ & 2.1 & $7(7.6 \%)$ & 3.3 & 0.02 \\
\hline PIH & $6(4.2 \%)$ & 0.9 & $26(4.9 \%)$ & $31(13.2 \%)$ & 2.8 & $15(17.6 \%)$ & 3.8 & $<0.0001$ \\
\hline PRE-ECLAMPSIA & $4(2.5 \%)$ & 0.7 & $23(4.3 \%)$ & $20(8.2 \%)$ & 2 & $11(12.8 \%)$ & 3 & 0.01 \\
\hline ANAEMIA & $83(57.3 \%)$ & 1.6 & $194(36.5 \%)$ & $41(17.1 \%)$ & 0.5 & $10(11.6 \%)$ & 0.3 & $<0.0001$ \\
\hline
\end{tabular}

Obese women had the highest incidence and risk of GDM, PIH, Pre-eclampsia whereas underweight women had the highest incidence and risk of anemia when compared to the other BMI group

Table 2: Table Showing The Incidence And Relative Risk Of Labour Outcomes And Early Puerperal Outcomes In Various Categories Of BMI:

\begin{tabular}{|l|l|l|l|l|l|l|l|l|}
\hline OUTCOMES & UNDER WEIGHT & RR & NORMAL WEIGHT & OVER WEIGHT & RR & OBESE & RR & p VALUE \\
\hline SVD & $74(51.1 \%)$ & - & $327(61.6 \%)$ & $106(44.7 \%)$ & - & $33(38.4 \%)$ & - & $<0.0001$ \\
\hline IVD & $4(3 \%)$ & 1.3 & $27(5 \%)$ & $20(8.2 \%)$ & 1.7 & $8(9.3 \%)$ & 1.9 & $<0.05$ \\
\hline LSCS & $66(45.9 \%)$ & 1.3 & $178(33.4 \%)$ & $112(47.1 \%)$ & 1.4 & $45(52.3 \%)$ & 1.6 & $<0.0001$ \\
\hline PPH & $10(7.1 \%)$ & 1.2 & $32(6 \%)$ & $29(12 \%)$ & 2 & $16(18.1 \%)$ & 3.1 & 0.0002 \\
\hline DWH & $3(1.7 \%)$ & 0.9 & $12(2.2 \%)$ & $13(5.6 \%)$ & 2.4 & $9(10.4 \%)$ & 4.1 & 0.0006 \\
\hline
\end{tabular}

Obese women had the highest incidence and risk of IVD, LSCS, PPH and DWH when compared to other BMI groups.

Table 3: Table Showing The Incidence And Relative Risk Of Neonatal Outcomes In Various Categories Of BMI:

\begin{tabular}{|l|l|l|l|l|l|l|l|l|}
\hline OUTCOMES & UNDER WEIGHT & RR & NORMAL EIGHT & OVER WEIGHT & RR & OBESE & RR & p VALUE \\
\hline LGA & $0 \%$ & - & $19(3.5 \%)$ & $14(5.9 \%)$ & 1.6 & $10(12 \%)$ & 3.2 & 0.0002 \\
\hline SGA & $30(21 \%)$ & 2.7 & $40(7.5 \%)$ & $19(7.8 \%)$ & 1.1 & $8(9.5 \%)$ & 1.2 & $<0.0001$ \\
\hline NICU ADMISSION & $6(4.4 \%)$ & 1.2 & $20(3.8 \%)$ & $16(6.9 \%)$ & 1.8 & $10(11.9 \%)$ & 3.2 & 0.01 \\
\hline
\end{tabular}

Out of the total 1000 cases, 9 babies delivered were stillborn and NICU admissions in 991 patients were studied. Obese women delivered the highest number of LGA babies and highest NICU admission whereas underweight women delivered the highest number of SGA babies.

\section{DISCUSSION:}

An attempt was made to compare the current study with those by various authors. BMI distribution of the study population is comparable to the study conducted by Anjana Verma et al[5]. In the present study, obese women had the highest incidence (7.6\%) and highest risk (3.3 times) of GDM ( $p=0.02)$. Similar results were found by Hamideh Pakniat et al [6] with GDM $(5.5 \%)(\mathrm{p}<0.001)$. In my study, obese women had the highest incidence (17.6\%) and highest risk (3.8 times) of PIH $(p<0.0001)$. Similar results were found by Shazia Shaukat et al [7] with PIH (12.2\%) in obese women ( $p=0.03)$. In my study, obese women had the highest incidence (12.8\%) and highest risk (3 times) of preeclampsia $(p=0.01)$. Similar results were found by Hamideh Pakniat et al [6] with preeclampsia (9.6\%) $(p=0.001)$ in obese women.

In my study, underweight women had the highest incidence $(57.3 \%)$ and highest risk (1.6 times) of anaemia $(p<0.0001)$, and obese women had the highest incidence (9.3\%) and highest risk ( 1.9 times) instrumental vaginal delivery $(p<0.05)$. Similar results were found by Anjana Verma et al [5] with anaemia $(58.6 \%)$ in underweight women $(p<0.001)$, instrumental delivery $(2.3 \%)$ in obese women $(p=0.86)$. As $\mathrm{GMCH}$, Guwahati is a tertiary care hospital, more number of complicated cases are being referred here which explains the increased instrumental delivery rates in our hospital. In my study, obese women had the highest incidence (52.3\%) and highest risk ( 1.6 times) of LSCS $(p<0.0001)$. Meenakshi et al [8] found similar results with LSCS (52.5\%) in obese women $(p<0.01)$. The present study showed that obese women had the highest incidence $(18.1 \%)$ and highest risk (3.1 times) of PPH $(\mathrm{p}=0.0002)$. Tharahalli et al [9] showed similar results with PPH $(12 \%)$ in obese women $(\mathrm{p}<0.001)$. In the present study, obese women had the highest incidence (10.4\%) and highest risk (4.1 times) of delayed wound healing $(p=0.0006)$. Similar results were found by Leticia Vieira de Paiva et al [10] with surgical wound infections $(16.7 \%)$ in obese women $(\mathrm{p}=0.04)$

In the present study, obese women had the highest incidence (12\%) and highest risk (3.2 times) of delivering LGA babies $(\mathrm{p}=0.0002)$, underweight women had the highest incidence $(21 \%)$ and highest risk (2.7 times) of delivering SGA babies $(p<0.0001)$ and babies born to obese women had the highest incidence $(10.9 \%)$ and highest risk (3.2 times) of NICU admission $(p<0.05)$. Similar results were given by Anjana Verma et al [5] with LGA babies (10.7\%) in obese women $(p<0.001)$ and SGA babies $(18.9 \%)(p<0.001)$ in underweight women and 9.5\% NICU admission $(p=0.03)$ in obese women.

\section{CONCLUSION:}

Various adverse feto-maternal outcomes are associated with both the extremes of BMI. Counselling all pregnant women about healthy and balanced diet and the potential risks and outcomes associated with the extremes of BMI is of utmost importance. Interventions to optimise the nutritional status and health of mothers, proper care and availability of health services and timely diagnosis of the complications help to reduce the adverse outcomes.

\section{Conflict of Interest: None}

\section{REFERENCES:}

1. WHO recommendations on health promotion interventions for maternal and 
newborn health. Geneva: World Health Organization; 2015. Available at: www.who.int/reproductivehealth/publications/maternal_perinatal_health/a nc-positive-pregnancy-experience/en/

2. Agrawal S \& Singh, A. (2016) Obesity or Underweight-What is Worse in Pregnancy? Journal of Obstetrics and Gynecology of India, 66(6), 448-452. https://doi.org/10.1007/s13224-015-0735-4

3. Triunfo, S., \& Lanzone, A. (2014). Impact of overweight and obesity on obstetric outcomes. Journal of Endocrinological Investigation, 37(4), 323-329.

4. Nuttall, F. Q. (2015). Body mass index: Obesity, BMI, and health: A critical review. Nutrition Today, 50(3), 117-128.

5. Verma, A., \& Shrimali, L. (2012). Maternal body mass index and pregnancy outcome. Journal of Clinical and Diagnostic Research, 6(9), 1531-1533.

6. Dr.mohammadi, fatemeh. (2015). The Impact of Body Mass Index on Pregnancy Outcome. Journal of Midwifery \& Reproductive Health, Md.

7. Shaukat S \& Nur U (2019). Effect of prepregnancy maternal BMI on adverse pregnancy and neonatal outcomes: Results from a retrospective cohort study of a multiethnic population in Qatar. BMJ Open, 9(9), 1-11.

8. Meenakshi, Reena, S., Rai, S. N., Kushwaha, K. P., \& Vani, A. (2012). Obstetric behavior and pregnancy outcome in overweight and obese women: Maternal and fetal complications and risks in relation to maternal overweight and obesity. Journal of Obstetrics and Gynecology of India, 62 (3), 276-280.

9. Paiva, L. V. de, Nomura, R. M. Y., Dias, M. C. G., \& Zugaib, M. (2012). Maternal obesity in high-risk pregnancies and postpartum infectious complications. Revista Da Associação Médica Brasileira (English Edition), 58(4), 453-458.

10. Tharihalli, C., \& Thathagari, V. (2016). Study of correlation between maternal body mass index with maternal and perinatal outcome. International Journal of Reproduction, Contraception, Obstetrics and Gynecology, 6(1), 164. 